\title{
Clinical Outcome and Prognostic Factors of Intensity-Modulated Radiotherapy for T4 Stage Nasopharyngeal Carcinoma
}

\author{
Yangkun Luo, ${ }^{1}$ Yang Gao, ${ }^{2}$ Guangquan Yang, ${ }^{1}$ and Jinyi Lang ${ }^{1}$ \\ ${ }^{1}$ Department of Radiation Oncology, Sichuan Cancer Hospital, Chengdu 610041, China \\ ${ }^{2}$ Department of Radiation Oncology, Zigong No. 4 People's Hospital of Sichuan Province, Zigong 643000, China \\ Correspondence should be addressed to Jinyi Lang; langjy610@163.com
}

Received 12 December 2015; Revised 27 March 2016; Accepted 31 March 2016

Academic Editor: Xin-yuan Guan

Copyright (c) 2016 Yangkun Luo et al. This is an open access article distributed under the Creative Commons Attribution License, which permits unrestricted use, distribution, and reproduction in any medium, provided the original work is properly cited.

\begin{abstract}
Objective. To analyze the clinical outcomes and prognostic factors of intensity-modulated radiotherapy (IMRT) for T4 stage nasopharyngeal carcinoma (NPC). Methods. Between March 2005 and March 2010, 110 patients with T4 stage NPC without distant metastases were treated. All patients received IMRT. Induction and/or concurrent chemotherapy were given. 47 (42.7\%) patients received IMRT replanning. Results. The 5-year local recurrence-free survival (LRFS), regional recurrence-free survival (RRFS), distant metastasis-free survival (DMFS), progression-free survival (PFS), and overall survival (OS) rates were 90.1\%, 97.0\%, 67.5\%, $63.9 \%$, and $64.5 \%$, respectively. Eleven patients experienced local-regional failure and total distant metastasis occurred in 34 patients. 45 patients died and 26 patients died of distant metastasis alone. The 5 -year LRFS rates were $97.7 \%$ and $83.8 \%$ for the patients that received and did not receive IMRT replanning, respectively $(P=0.023)$. Metastasis to the retropharyngeal lymph nodes (RLN) was associated with inferior 5 -year OS rate $(61.0 \%$ versus $91.7 \%, P=0.034)$. The gross tumor volume of the right/left lymph nodes (GTVln) was an independent prognostic factor for DMFS $(P=0.006)$ and PFS $(P=0.018)$. GTVln was with marginal significance as the prognostic factor for OS $(P=0.050)$. Conclusion. IMRT provides excellent local-regional control for T4 stage NPC. Benefit of IMRT replanning may be associated with improvement in local control. Incorporating GTVln into the N staging system may provide better prognostic information.
\end{abstract}

\section{Introduction}

Nasopharyngeal carcinoma (NPC) is a common malignancy in southern China and radiotherapy (RT) is the primary treatment [1]. The five-year overall survival (OS) rate for early-stage NPC has exceeded $90 \%$ following treatment with RT alone [2]. However, it is important to note that the majority of patients present with locoregionally advanced stages of NPC at the time of diagnosis. According to the American Joint Committee on Cancer (AJCC) 2010 staging system [3], T4 stage NPC is characterized as a tumor with intracranial extensions and/or involvement of the cranial nerves, hypopharynx, orbit, and extensions to the infratemporal fossa/masticator space. RT of T4 stage NPC represents a great challenge due to dose limitation requirements for critical structures such as the spinal cord and brainstem.

Intensity-modulated radiotherapy (IMRT) has replaced two-dimensional RT due to its dosimetric advantages. The use of IMRT and chemotherapy in combination has also been found to provide excellent locoregional control [46]. Unfortunately, however, improvement in locoregional control has not been accompanied by an increase in longterm OS. While studies of concurrent chemoradiation [7-11] which have included different combinations of chemotherapy and radiation have shown high rates of locoregional control, OS and particularly distant metastasis-free survival (DMFS) rates remain suboptimal. Furthermore, data regarding the clinical outcome of T4 stage NPC cases are relatively rare.

Adaptive radiotherapy (ART) has also been studied in the setting of IMRT for head and neck cancers. For this treatment approach, RT plan is modified in response to anatomic and dosimetric changes during treatment in order to ensure adequate target coverage and to improve quality of life by reducing the treatment dose to normal tissue [12-16]. To date, many studies have focused on dosimetric analyses, and limited data have been published regarding the effect of ART 
on clinical outcome. In the present study, treatment results from cases of T4 stage NPC were retrospectively analyzed and both clinical outcome and prognostic factors were examined. In addition, the potential clinical benefit of adaptive IMRT replanning is discussed.

\section{Materials and Methods}

2.1. Pretreatment Evaluations of the Patients Selected. Between March 2005 and March 2010, 110 patients that were newly diagnosed with T4 stage NPC and were treated at the Sichuan Cancer Hospital were enrolled in this study. The pretreatment workup performed for each patient included a complete history and physical examination, complete blood counts, blood chemistries, an endoscopy, magnetic resonance imaging (MRI) of the nasopharynx and neck, chest computed tomography (CT) or radiography, an abdominal ultrasound, and emission computed tomography (ECT). The inclusion criteria were as follows: (1) pathologically confirmed NPC, (2) receiving radical IMRT treatment, (3) a Karnofsky performance score > 70, (4) receiving chemotherapy, (5) complete RT plan documentation, (6) an absence of pregnancy or lactation, and (7) an absence of previous malignancy or other concomitant malignant disease. Patients with distant metastasis at diagnosis and those who did not complete a full course of RT were excluded. All patients underwent disease restaging according to the AJCC 2010 staging system [3] and patient characteristics for the present cohort are summarized in Table 1 . This study was approved by the ethics committee of Sichuan Cancer Hospital.

2.2. RT. All patients were treated with IMRT with 6megavoltage (MV) photons. Target volumes were in agreement with the International Commission on Radiation Units and Measurements Reports 50 and 62 [17, 18]. RT planning was designed and optimized using the CORVUS 3.4-4.2 inverse treatment planning system (Peacock, Nomos, Deer Park, IL, USA). The gross tumor volume of the nasopharynx (GTVnx) and gross tumor volume of the right/left lymph nodes (GTVln) were outlined based on CT and MRI scans. Clinical target volume 1 (CTV1) included the GTVnx with a $5-10 \mathrm{~mm}$ margin and high risk structures. Clinical target volume 2 (CTV2) potentially involved regions including the nasopharyngeal cavity, the maxillary sinus, the pterygopalatine fossa, the posterior ethmoid sinus, the parapharyngeal space, the skull base, the anterior third of the clivus, the inferior sphenoid sinus, and the cavernous sinus. The clinical target volume of the right/left lymph nodes (CTVln) included the lymphatic drainage regions (the bilateral retropharyngeal nodes and levels II, III, and $\mathrm{V}_{\mathrm{A}}$ ). The prescribed radiation doses were defined as follows: 66-76 Gy for GTVnx, 6070 Gy for GTVln, 60-66 Gy for CTV1, 54-60 Gy for CTV2, and 50-54 Gy for CTVln using a simultaneous integrated boost technique, each divided into 30-33 deliveries. In addition, the dose limits for each normal organ were set according to the Radiation Therapy Oncology Group protocol 0225 [5]. The prescribed dose encompassed at least $95 \%$ of the target
TABLE 1: Characteristics of 110 patients with T4 stage NPC.

\begin{tabular}{|c|c|}
\hline Patient characteristics & Number of patients (\%) \\
\hline \multicolumn{2}{|l|}{ Age (y) } \\
\hline Range & $17-69$ \\
\hline Median & 44.5 \\
\hline \multicolumn{2}{|l|}{ Gender } \\
\hline Male & $87(79.1)$ \\
\hline Female & $23(20.9)$ \\
\hline \multicolumn{2}{|l|}{$\mathrm{N}$ stage } \\
\hline N0-1 & $35(31.8)$ \\
\hline N2-3 & $75(68.2)$ \\
\hline \multicolumn{2}{|l|}{ GTVnx (cc) } \\
\hline Range & $22.37-246.10$ \\
\hline Median & 77.27 \\
\hline \multicolumn{2}{|l|}{ GTVln (cc) } \\
\hline Range & $0-180.15$ \\
\hline Median & 14.1 \\
\hline \multicolumn{2}{|l|}{ IMRT replanning } \\
\hline No & $63(57.3)$ \\
\hline Yes & $47(42.7)$ \\
\hline \multicolumn{2}{|l|}{ Chemotherapy } \\
\hline Concurrent & $75(68.2)$ \\
\hline Induction plus concurrent & $35(31.8)$ \\
\hline \multicolumn{2}{|l|}{ Targeted therapy } \\
\hline No & $83(75.5)$ \\
\hline Yes & $27(24.5)$ \\
\hline \multicolumn{2}{|l|}{ Metastasis to RLN } \\
\hline No & $13(11.8)$ \\
\hline Yes & $97(88.2)$ \\
\hline \multicolumn{2}{|l|}{ Cervical nodal necrosis } \\
\hline No & $68(61.8)$ \\
\hline Yes & $42(38.2)$ \\
\hline
\end{tabular}

GTVnx, gross tumor volume of the nasopharynx; GTVln, gross tumor volume of the lymph nodes; IMRT, intensity-modulated radiotherapy; RLN, retropharyngeal lymph nodes.

volume, and no greater than $1 \%$ of the nasopharynx gross target volume received $\leq 93 \%$ of the prescribed dose. The maximum dose of the treatment plan was applied within the target volume.

The IMRT plan was applied via dynamic intensitymodulated coplanar arc irradiation using a MIMI multileaf collimator (NOMOS Corporation, Sewickley, PA, USA). For all of the patients, radiation was applied to the lymph node drainage areas in the lower neck by using ${ }^{60}$ Co split field techniques or $6 \mathrm{MV} \mathrm{X}$-ray split-beam techniques, with a prescription dose of $50 \mathrm{~Gy}$ in 25 fractions. Positive cervical lymph nodes in the lower neck received a total dose of 60$70 \mathrm{~Gy}$ by electron beam boost irradiation.

For this study, target volumes (including GTVnx and GTVln) were measured by the treatment planning system. The system automatically calculated the volume by the summation of area technique. Because the split-beam technique was used in this study, the low neck positive cervical lymph 
nodes were not included in the initial IMRT plan. These positive cervical lymph nodes were contoured according to the description of the boost plan. Then the whole volumes of positive cervical lymph nodes were recalculated by the system.

2.3. IMRT Replanning. A total of 47 (42.7\%) patients received 1-4 IMRT replans (median: 3). The decision to replan was made at the physician's discretion and multiple factors were considered: weight loss, nutritional status, changes in palpable or visible tumor size, an ill-fitting mask, and the extent of acute radiation reactions. When a tumor was close to the spinal cord or brainstem and other important organs, replanning was typically needed early in the intervention process, and additional replans could be made if needed. If a dose escalation was needed, a replan was routinely administered and a new CT scan would be performed. During each CT scan, the patient maintained the same position and the new CT scan was used to generate a new IMRT plan for the corresponding fractions of treatment. To ensure relative consistency of target delineation, a CT-CT fusion was used by rigid registration and was adjusted manually according to the region of interest. GTVnx and GTVln, along with the organs at risk, were contoured on the new CT scans. The CTV was maintained and modified according to the changes in anatomic structure that occurred. The time from resimulation to implementation of the new IMRT plan was generally 1-3 days. The first IMRT replan was implemented at a median dose of $44 \mathrm{~Gy}$ (range, 8.8-57.2).

2.4. Chemotherapy. All patients received platinum-based chemotherapy and did not receive adjuvant chemotherapy. The induction chemotherapy regimens included TP $\left(75 \mathrm{mg} / \mathrm{m}^{2}\right.$ docetaxel and $80 \mathrm{mg} / \mathrm{m}^{2}$ cisplatin on day 1 ) or PF $\left(100 \mathrm{mg} / \mathrm{m}^{2}\right.$ cisplatin on day 1 and $1000 \mathrm{mg} / \mathrm{m}^{2} /$ day 5 -fluorouracil for days 1-5) every three weeks for 1-2 cycles. Concurrent chemotherapy included $80 \mathrm{mg} / \mathrm{m}^{2}$ cisplatin every three weeks for 2-3 cycles. A total of 27 (24.5\%) patients received targeted therapy including cetuximab or nimotuzumab.

2.5. Patient Assessments and Follow-Up. All patients were evaluated weekly during RT and then they underwent followup upon completion of RT, as well as 1 month after the completion of RT, every 3 months in the first 2 years, every 6 months from year 3 to year 5, and annually thereafter. Each follow-up included a complete examination involving a flexible fiberoptic endoscopy, an ultrasound of the abdomen, a chest X-ray, and basic serum chemistry. Either CT or MRI of the head and neck was performed after the completion of IMRT and then was performed every 6 months from then on. Treatment-related toxicities were assessed according to the National Cancer Institute Common Toxicity Criteria (NCI-CTC) version 3.0 [19]. RT-related toxicities were graded according to RTOG criteria [20].

2.6. Statistical Analysis. All analyses were performed with SPSS, version 16.0 (IBM, Chicago, IL, USA). Local recurrence-free survival (LRFS), regional recurrence-free survival

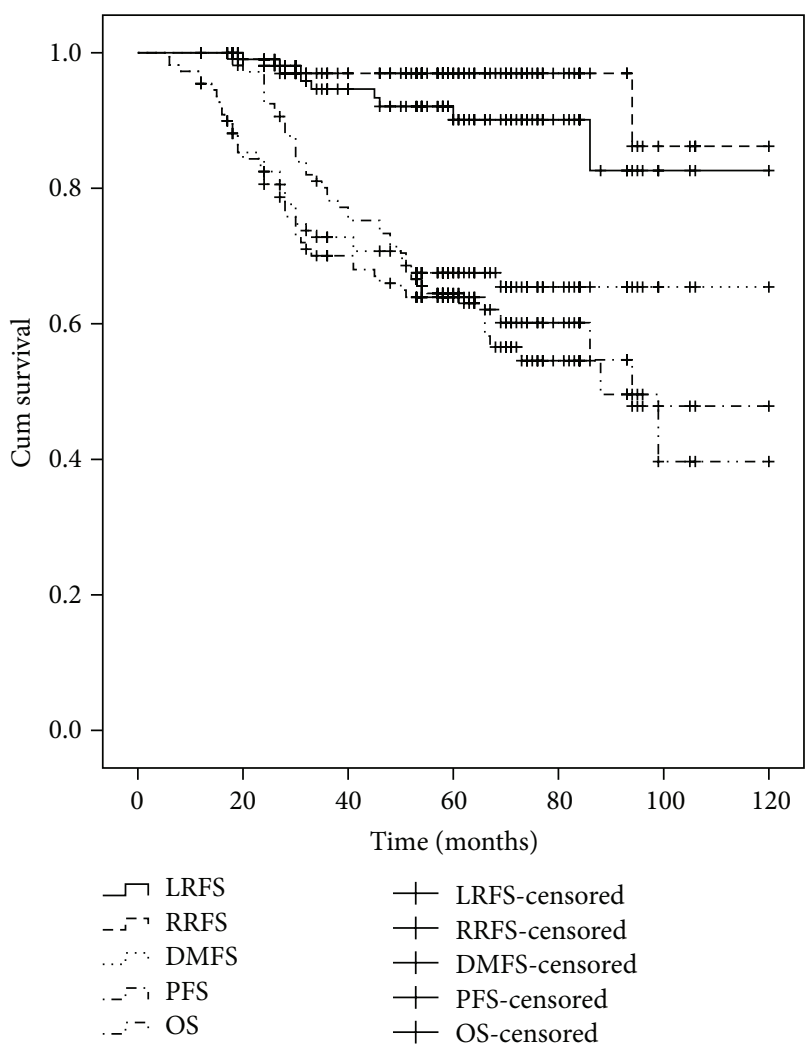

FIGURE 1: Kaplan-Meier curves for LRFS, RRFS, DMFS, PFS, and OS for the present cohort.

(RRFS), DMFS, progression-free survival (PFS), and OS were estimated according to the Kaplan-Meier method. LRFS, RRFS, DMFS, PFS, and OS were measured from day 1 of treatment to the date of the event. For the univariate and multivariate analyses performed, the log-rank test and the Cox proportional hazards model were employed, respectively. A $P$ value less than 0.05 was considered to be statistically significant.

\section{Results}

3.1. Treatment Outcome. The median follow-up time for the present cohort was 58 months (range, 12-120 months). The overall 5-year LRFS, RRFS, DMFS, PFS, and OS rates were $90.1 \%, 97.0 \%, 67.5 \%, 63.9 \%$, and 64.5\%, respectively (Figure 1).

The 5-year LRFS rates for patients with or without IMRT replanning were $97.7 \%$ and $83.8 \%$, respectively $(P=0.023)$ (Figure 2(a)). In addition, the 5-year DMFS, PFS, and OS rates for the patients with or without IMRT replanning were $71.1 \%$ and $65.9 \%, 69.4 \%$ and $56.7 \%$, and $67.2 \%$ and $62.6 \%$, respectively, in each case (Table 2 ).

The 5-year OS rates for the patients with or without metastasis to the retropharyngeal lymph nodes (RLN) were $61.0 \%$ and $91.7 \%$, respectively $(P=0.034)$ (Figure $2(\mathrm{~b}))$. The 5 -year DMFS, PFS, and OS for patients with GTVln $\leq 14.1 \mathrm{cc}$ and GTVln > 14.1cc were 79.0\% and 56.1\% $(P=0.007)$, $73.1 \%$ and $55.0 \%(P=0.021)$, and $75.6 \%$ and $53.0 \%(P=$ 


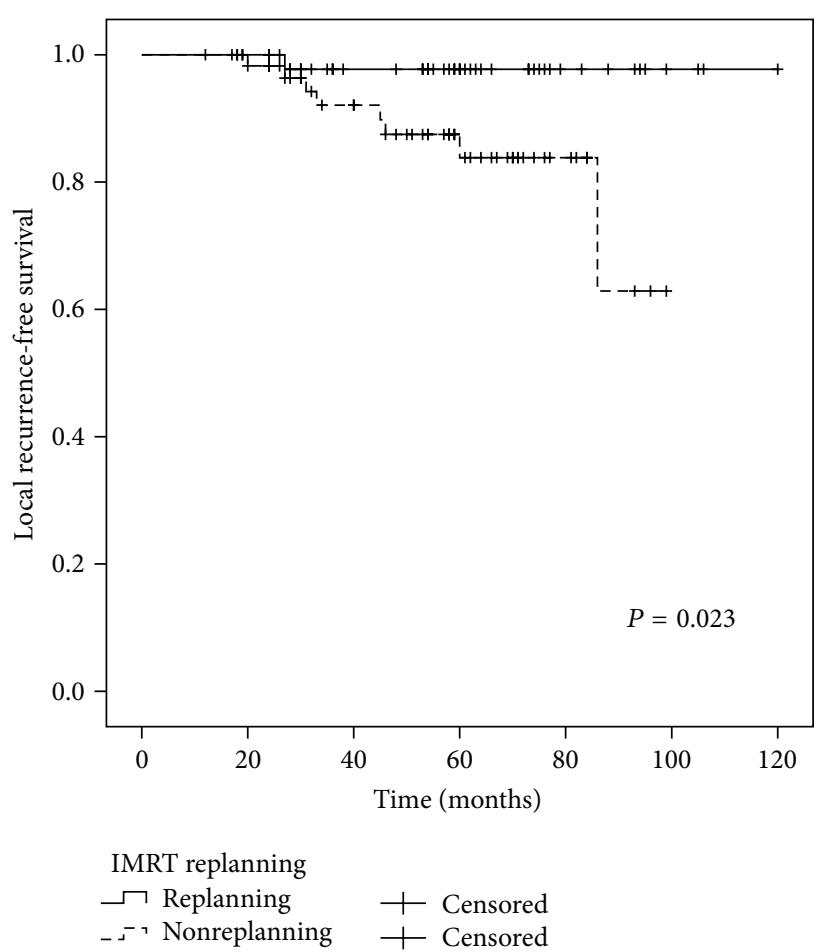

(a)

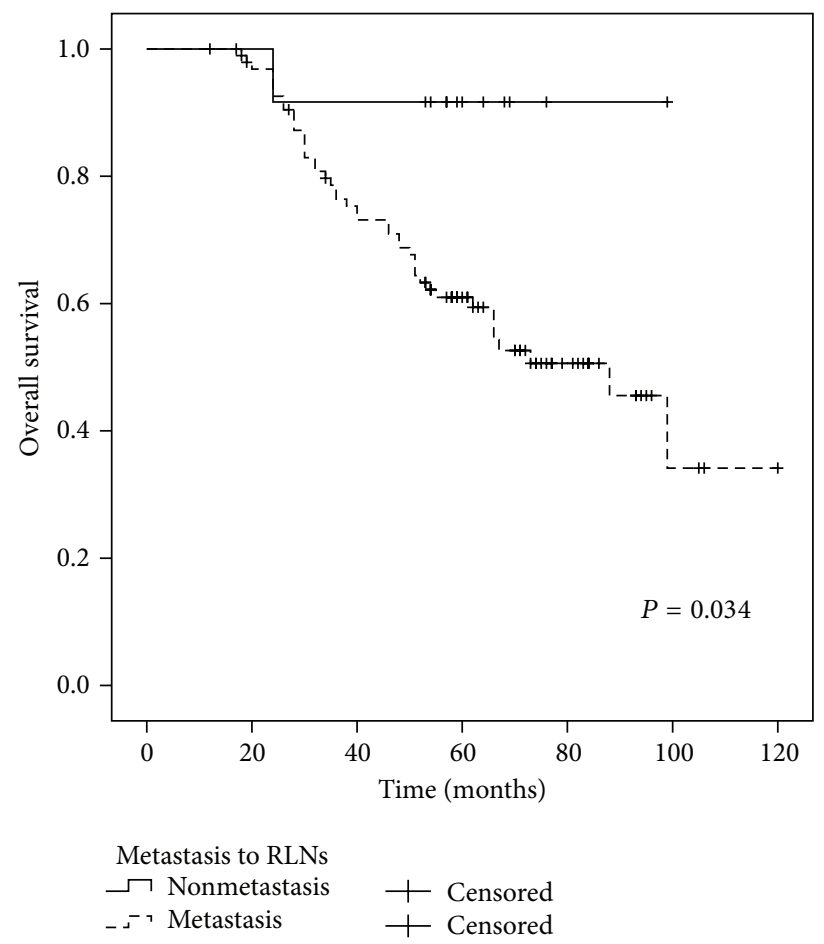

(b)

FIGURE 2: (a) Comparison of the 5-year LRFS curves for the patients that were treated with and without IMRT replanning. Log-rank test; $P=0.023$. (b) Comparison of the 5-year OS curves for the patients with or without metastasis to the RLN. Log-rank test; $P=0.034$.

0.028), respectively (Figures 3-5). There were no statistically significant differences in the LRFS, RRFS, DMFS, and OS rates in the GTVnx, cervical nodal necrosis, targeted therapy, and induction or concurrent chemotherapy. A total of 45 patients died due to severe bleeding of the nasopharynx $(n=5)$, severe bleeding of the nasopharynx combined with locoregional recurrence $(n=1)$, unknown internal disease $(n=5)$, locoregional recurrence $(n=5)$, locoregional recurrence with distant metastasis $(n=3)$, and distant metastasis $(n=26)$. Furthermore, a total of 41 failures were observed that involved distant metastasis alone $(n=30)$, local recurrence alone $(n=5)$, cervical lymph node relapse alone $(n=2)$, local recurrence and distant metastasis $(n=3)$, and cervical lymph node relapse and distant metastasis $(n=1)$.

3.2. Prognostic Factors. The value of various potential prognostic factors, including age, gender, N stage, GTVnx, GTVln, chemotherapy, IMRT replanning, targeted therapy, metastasis to the RLN, and cervical nodal necrosis, for predicting LRFS, RRFS, DMFS, PFS, and OS was evaluated. Univariate analysis showed that GTVln was significantly associated with DMFS, PFS, and OS, while IMRT replanning was significantly associated with LRFS.

There was no difference in DMFS, PFS, and OS for the patients with or without IMRT replanning $(P=0.743, P=$ 0.302 , and $P=0.646$, resp.) (Table 2 ).

Furthermore, metastasis to the RLN was significantly associated with OS (Table 2).
In multivariate analysis, GTVln, IMRT replanning or not, and metastasis to the RLN or not which were proved by univariate analysis to have the potential affecting survival rate were included in the Cox proportional hazard model. The results showed that GTVln was an independent negative prognostic factor of DMFS and PFS $(\mathrm{HR}=2.718,95 \% \mathrm{CI}$ : 1.324-5.577, and $P=0.006$ and $\mathrm{HR}=2.159,95 \% \mathrm{CI}: 1.141-$ 4.088 , and $P=0.018$, resp.). The GTVln also exhibited marginal significance as a prognostic factor for OS $(\mathrm{HR}=$ 1.875, 95\% CI: $1.001-3.512$, and $P=0.050)$.

3.3. Toxicity. All patients tolerated the entire treatment regimen. The main manifestations of radiation-related acute toxicity included xerostomia, mucositis, and dermatitis. The acute toxicities were controllable and were mainly characterized as grades 1-2. However, grade 3 xerostomia, mucositis, and dermatitis were observed, with rates of $4.5 \%(n=$ 5), $22.7 \%(n=25)$, and $6.4 \%(n=7)$, respectively. None of the patients experienced grade 4 toxicity. Regarding hematologic toxicity, 10 (9.1\%) patients experienced grade 4 leukopenia, while liver and kidney dysfunction were mainly grades 1-2. Late onset toxicities were assessed for all of the 110 patients at least one year after the completion of treatment. The incidence of grades $0-1,2,3$, and 4 xerostomia was 73.6\% $(n=81), 23.6 \%(n=26), 2.7 \%(n=3)$, and $0.0 \%$, respectively. Only one patient that underwent IMRT replanning developed grade 3 xerostomia, and there were no cases of radiation-induced cranial nerve palsy. There were 
TABLE 2: Impact of prognostic factors on treatment according to univariate analysis (log-rank test).

\begin{tabular}{|c|c|c|c|c|c|c|c|c|c|c|}
\hline Characteristics & LRFS & $P$ & RRFS & $P$ & DMFS & $P$ & PFS & $P$ & OS & $P$ \\
\hline \multicolumn{11}{|l|}{ Age (yrs) } \\
\hline$\leq 44.5 \mathrm{yrs}$ & 89.4 & \multirow{2}{*}{0.743} & 96.0 & \multirow{2}{*}{0.895} & 62.4 & \multirow{2}{*}{0.418} & 63.9 & \multirow{2}{*}{0.876} & 63.5 & \multirow{2}{*}{0.813} \\
\hline$>44.5 \mathrm{yrs}$ & 90.4 & & 98.0 & & 73.1 & & 63.8 & & 65.4 & \\
\hline \multicolumn{11}{|l|}{ Gender } \\
\hline Male & 90.0 & \multirow{2}{*}{0.334} & 96.2 & \multirow{2}{*}{0.331} & 62.4 & \multirow{2}{*}{0.418} & 63.9 & \multirow{2}{*}{0.876} & 63.0 & \multirow{2}{*}{0.493} \\
\hline Female & 91.1 & & 100.0 & & 73.1 & & 63.8 & & 69.6 & \\
\hline \multicolumn{11}{|l|}{$\mathrm{N}$ stage } \\
\hline N0-1 & 91.9 & \multirow{2}{*}{0.807} & 100.0 & \multirow{2}{*}{0.110} & 75.8 & \multirow{2}{*}{0.339} & 75.9 & \multirow{2}{*}{0.272} & 79.4 & \multirow{2}{*}{0.087} \\
\hline $\mathrm{N} 2-3$ & 89.4 & & 95.6 & & 64.1 & & 58.7 & & 57.3 & \\
\hline \multicolumn{11}{|l|}{ GTVnx (cc) } \\
\hline$\leq 77.27$ & 85.7 & \multirow{2}{*}{0.161} & 97.8 & \multirow{2}{*}{0.728} & 68.4 & \multirow{2}{*}{0.386} & 61.1 & \multirow{2}{*}{0.274} & 66.6 & \multirow{2}{*}{0.584} \\
\hline$>77.27$ & 95.4 & & 96.1 & & 70.3 & & 67.0 & & 62.4 & \\
\hline \multicolumn{11}{|l|}{ GTVln (cc) } \\
\hline$\leq 14.1$ & 89.5 & \multirow{2}{*}{0.693} & 98.1 & \multirow{2}{*}{0.382} & 79.0 & \multirow{2}{*}{0.007} & 73.1 & 0.021 & 75.6 & 0.028 \\
\hline$>14.1$ & 91.3 & & 95.7 & & 56.1 & & 55.0 & 0.021 & 53.0 & 0.028 \\
\hline Chemotherapy & & & & & & & & & & \\
\hline Concurrent & 90.8 & 0788 & 97.0 & 0431 & 71.6 & 0214 & 68.6 & 0149 & 70.2 & 0250 \\
\hline Induction plus concurrent & 89.3 & 0.100 & 97.0 & .401 & 58.8 & 0.217 & 54.1 & 0.14 & 52.6 & 0.250 \\
\hline IMRT replanning & & & & & & & & & & \\
\hline No & 83.8 & 0023 & 96.2 & 0.853 & 65.9 & 0.743 & 56.7 & 0.302 & 62.6 & 0.646 \\
\hline Yes & 97.7 & & 97.9 & & 71.1 & & 69.4 & & 67.2 & \\
\hline Targeted therapy & & & & & & & & & & \\
\hline No & 87.8 & 0308 & 97.6 & 0.339 & 69.6 & 0.532 & 64.7 & 0.941 & 62.6 & 0.841 \\
\hline Yes & 96.2 & 0.500 & 100.0 & & 61.5 & & 61.5 & & 70.0 & \\
\hline Metastasis to RLN & & & & & & & & & & \\
\hline No & 90.9 & 0.941 & 100.0 & 0.468 & 91.7 & 0.075 & 83.3 & 0.122 & 91.7 & 0.034 \\
\hline Yes & 90.1 & & 96.6 & & 64.3 & & 61.4 & & 61.0 & \\
\hline Cervical nodal necrosis & & & & & & & & & & \\
\hline No & 92.1 & 0181 & 97.0 & 0.660 & 73.7 & 0.065 & 71.0 & 0.064 & 71.6 & 0.112 \\
\hline Yes & 86.3 & & 96.7 & & 57.3 & & 52.0 & 0.004 & 51.6 & \\
\hline
\end{tabular}

13 patients that developed radiation-induced temporal lobe necrosis that was diagnosed by MRI during the follow-up period. Four of these patients $(4 / 47 ; 8.5 \%)$ underwent IMRT replanning and nine of these patients $(9 / 63 ; 14.3 \%)$ did not undergo IMRT replanning.

\section{Discussion}

IMRT has replaced two-dimensional RT for the treatment of NPC because it delivers a higher radiation dose to a tumor while sparing the adjacent organs at risk. This can potentially enhance treatment outcome, especially the local control rate. In a study by Chen et al. [21], the 5-year OS, local relapse-free, and DMFS rates for 140 patients with T4 stage NPC that were treated by IMRT were $69.3 \%, 84.9 \%$, and $73.6 \%$, respectively. In another study [22] of 335 T4 stage NPC patients, the 5year local failure-free survival (LFFS), regional failure-free survival (RFFS), distant failure-free survival (DFFS), and OS rates were $84.1 \%, 92.2 \%, 74.1 \%$, and $63.0 \%$, respectively. Sun et al. [6] reported that 5-year disease-specific survival (DSS), DMFS, LRFS, and PFS rates were 70.7\%, 71.7\%, 83.3\%, and 59.8\%, respectively. Similar 5-year rates for LRFS, RRFS, DMFS, PFS, and OS were observed in the present study (e.g., $90.1 \%, 97.0 \%, 67.5 \%, 63.9 \%$, and $64.5 \%$, resp.).

Distant metastasis was the main pattern of failure and the major cause of death in the present study. A total of 11 patients experienced local-regional failure and total distant metastasis occurred in 32 patients. There were 26 patients that died of distant metastasis alone. These results are similar to those reported by other studies of IMRT [5, 6, 23-25]. Therefore, although IMRT has been reported to provide excellent localregional control, an improvement in OS was not observed as well. It is well known that $\mathrm{N}$ stage is related to distant metastasis in NPC patients. Previously, N status was found to be a significant predictor for distant failure and survival [26]. N status usually considers the size of a node, the node level, extranodal neoplastic spread (ENS), nodal necrosis, metastasis to the RLN, and other factors [6, 26-29]. In the univariate analysis performed in the present study, metastasis to the RLN was significantly associated with OS $(P=0.034)$. Cervical nodal necrosis also exhibited a marginal value for DMFS and PFS $(P=0.065$ and $P=0.064$, resp. $)$ and $\mathrm{N}$ 


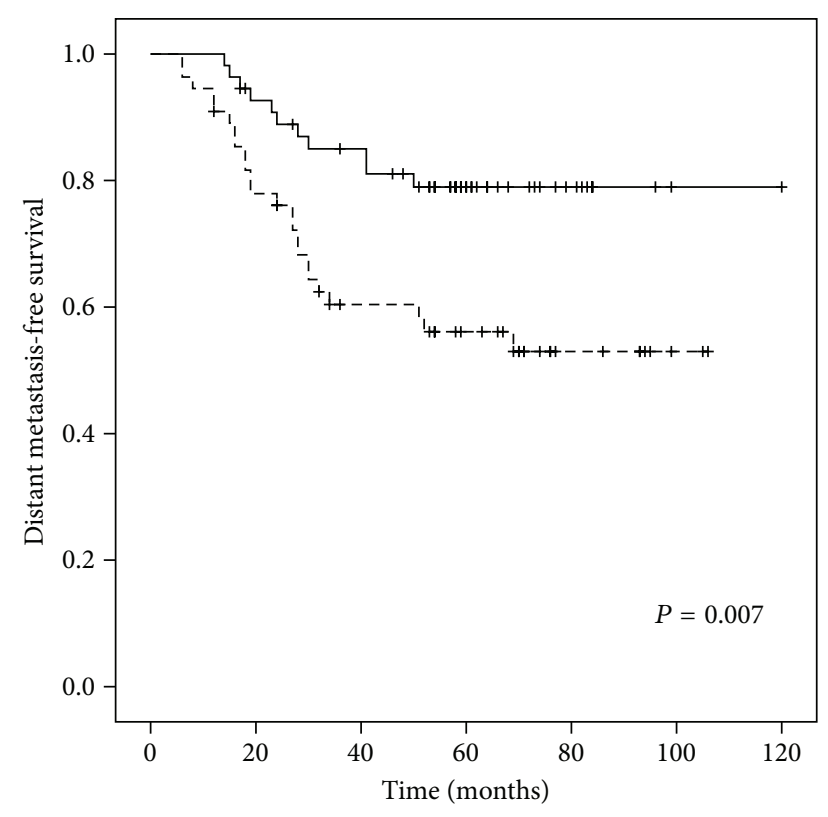

GTVln volume

$$
\begin{array}{ll}
\neg \text { Volume } \leq 14.1 c c \quad+\text { Censored } \\
-\neg \text { Volume }>14.1 c c \quad+\text { Censored }
\end{array}
$$

FIGURE 3: Comparison of the 5-year DMFS curves for the patients with a GTVln $\leq 14.1 \mathrm{cc}$ versus a GTVln $>14.1 \mathrm{cc}$. Log-rank test; $P=$ 0.007 .

stage was a marginal predictor for OS $(P=0.087)$. In the multivariate analysis, GTVln was an independent negative prognostic factor of DMFS and PFS $(P=0.006$ and $P=$ 0.018, resp.), while GTVln exhibited marginal significance as a prognostic factor for OS $(P=0.050)$. Based on these results, it would appear that tumor burden is more important than N stage alone. Thus, while IMRT can achieve excellent local control, it does not eliminate micrometastatic lesions that are present prior to treatment, and this is consistent with the high local control and low DMFS and OS rates that characterize this treatment regimen. Furthermore, based on the present results, incorporating GTVln into the $\mathrm{N}$ staging system for T4 stage NPC may increase the prognostic value of this parameter, and this should be further studied.

Severe bleeding of the nasopharynx is a fatal complication after radiotherapy. The reason may be related to nasopharynx necrosis. When the necrotic lesion invades carotid artery, especially internal carotid artery, leading to rupture of the artery wall, it can cause severe bleeding [30]. $T$ stage, radiation dose, and course of irradiation were the reasons of the nasopharynx necrosis [31]. In the present study, 5 patients died due to severe bleeding of the nasopharynx. For these patients, high total radiation dose (>70 Gy) and fraction dose ( $>2.2 \mathrm{~Gy}$ ) were given. Moreover, 2 patients with IMRT replanning were treated with higher radiation dose ( $\geq 74 \mathrm{~Gy})$. In addition, the parapharyngeal space was invaded and the internal carotid artery was enclosed by the primary tumor. These may lead to higher dose on the soft tissue and internal carotid artery, increased risk of necrosis, and severe bleeding

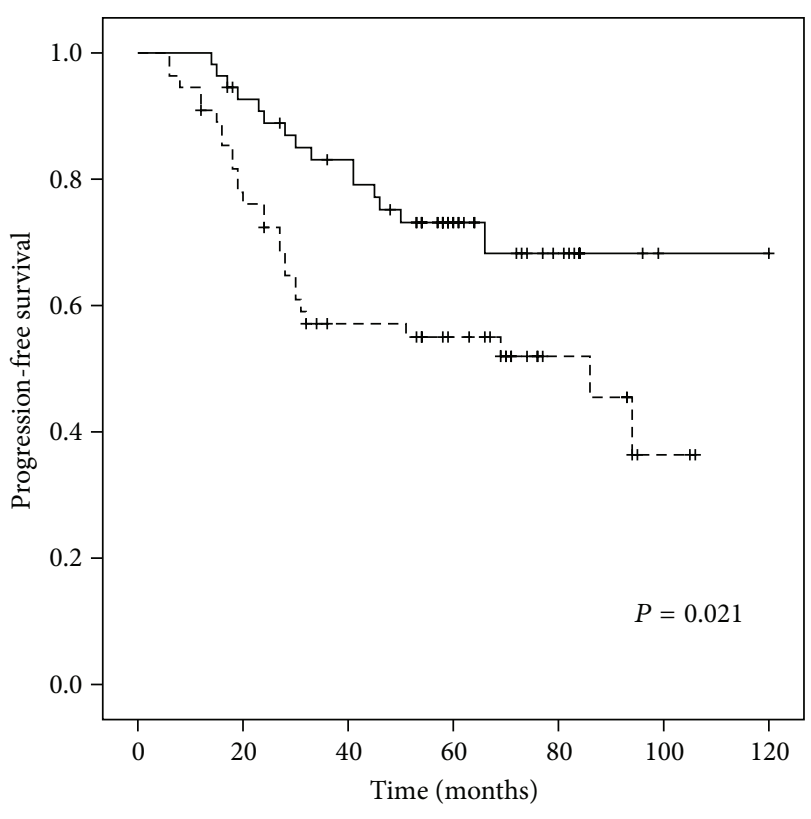

GTVln volume

$\neg$ Volume $\leq 14.1 \mathrm{cc} \quad$ Censored
$-\lrcorner$ Volume $>14.1 \mathrm{cc} \quad$ Censored

FIGURE 4: Comparison of the 5-year PFS curves for the patients with a GTVln $\leq 14.1$ cc versus a GTVln $>14.1$ cc. Log-rank test; $P=0.021$.

of the nasopharynx. So, more caution should be considered in the dose distribution for these patients.

Compared with conventional two-dimensional RT, IMRT has not improved distant control for $\mathrm{N}$ stage tumors. Thus, the survival benefits of IMRT for NPC patients may solely derive from improved local control [32]. In recent years, ART has been evaluated as an IMRT treatment. The purpose of ART is to ensure adequate target coverage and to improve quality of life by reducing the radiation dose that is applied to normal tissues [12-16]. To correct for variations in tumors and normal tissues, ART is used to modify RT plan at the corresponding time points based on the acquisition of online or offline images. In the present study, adaptive IMRT replanning was considered based on an offline image that was obtained at our hospital. The first IMRT replan was implemented with a median dose of $44 \mathrm{~Gy}$ (range, 8.8-57.2). There were a total of $47(42.7 \%)$ patients that received 14 IMRT replans (median: 3 ). While there was no standard for determining whether the replanning and the intervention time were successful, the results obtained show that IMRT replanning achieved better local control compared with nonIMRT replanning (97.7\% versus $83.8 \%$, resp.; $P=0.023$ ), yet the rates of DMFS, PFS, and OS were not statistically significant. The latter results are also consistent with those of previous studies $[12,13,16]$.

The treatment-related toxicities observed in the present study were well tolerated by the patients involved, and this has been observed in previous studies $[6,22]$. In particular, it was observed that the patients who underwent IMRT replanning had a lower incidence of temporal lobe necrosis 


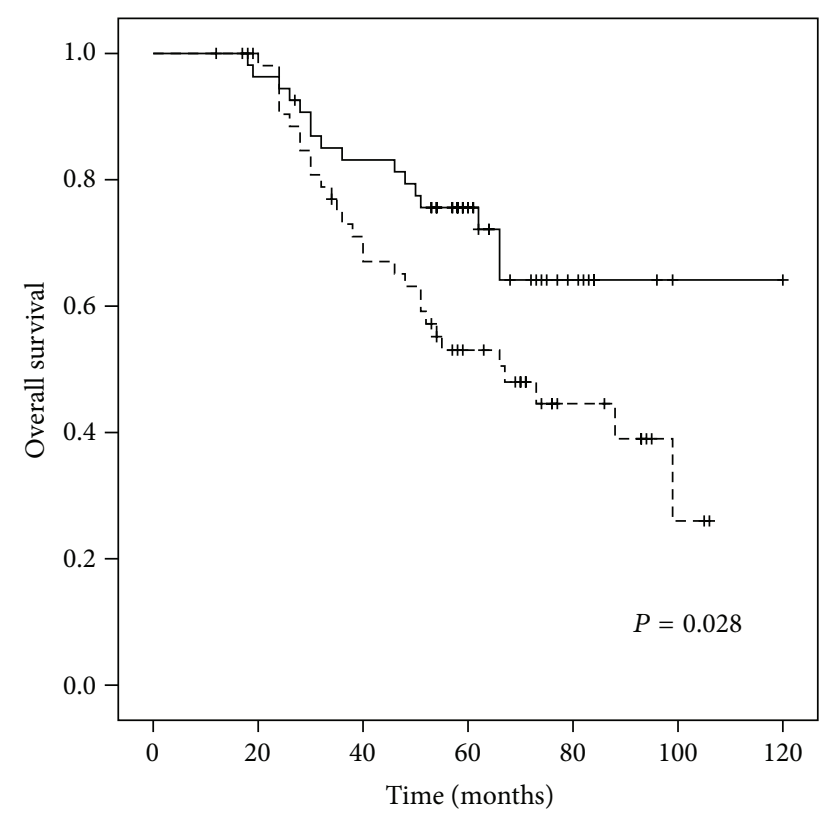

GTVln volume

$$
\begin{array}{ll}
\neg \text { Volume } \leq 14.1 \mathrm{cc} \quad+\text { Censored } \\
-\neg \text { Volume }>14.1 \mathrm{cc} \quad \text { Censored }
\end{array}
$$

FIGURE 5: Comparison of the 5-year OS curves for the patients with a GTVln $\leq 14.1$ cc versus a GTVln $>14.1$ cc. Log-rank test; $P=0.028$.

compared with the patients that did not undergo IMRT replanning ( $8.5 \%$ versus $14.3 \%$, resp.). However, a direct comparison is not entirely appropriate due to the imbalance in the distribution of patients that did or did not undergo IMRT replanning, although this result may partly explain the theory of the association of improved quality of life associated with IMRT replanning that was proposed by Yang et al. [13]. To our knowledge, the present study is one of the first studies to suggest that the benefits of adaptive IMRT replanning may be associated with an actual clinical advantage, including improved local control and late toxicities such as temporal lobe necrosis for T4 stage NPC patients. However, it is important to note that the present study is limited by a small sample size. Therefore, the benefit of IMRT replanning remains to be confirmed with a larger sample size. Furthermore, prospective randomized trials are needed to identify specific predictive factors and to determine the criteria for adaptive IMRT replanning for NPC patients.

Currently, the treatment for locoregional advanced NPC includes concurrent chemotherapy/RT with or without adjuvant chemotherapy and induction chemotherapy followed by chemotherapy/RT. It has been hypothesized that induction chemotherapy is able to target a greater number of local and distant metastasis tumor cells, while also decreasing radiation side effects by reducing tumor burden. In the present study, there was no statistically significant difference between induction chemotherapy and concurrent chemotherapy in relation to LRFS, RRFS, DMFS, PFS, and OS. There was also no statistically significant difference in the use of targeted therapy or not. However, in a recent meta-analysis [33], induction chemotherapy was found to significantly reduce the hazard of progression and distant metastasis in cases of local advanced NPC on the basis of concurrent chemoradiotherapy. Another treatment regimen for local advanced NPC involves concurrent chemo/RT followed adjuvant chemotherapy. In a Bayesian network meta-analysis [34], however, adjuvant chemotherapy did not appear to improve patient survival following chemoradiotherapy. Meanwhile, a MAC-NPC meta-analysis [35] demonstrated that the benefit of a concomitant plus adjuvant chemotherapy schedule produced the greatest tumor-related outcomes compared with other treatment modalities. Thus, the results for induction or adjuvant chemotherapy for the treatment of NPC vary and are contradictory. Therefore, it remains for the treatment modalities for T4 stage NPC to be further studied.

There are several limitations associated with the present study, including the retrospective nature of the study design and the small number of studied patients. Possible prognostic factors, such as involvement of cranial nerves and the extent of intracranial involvement, also were not studied because of insufficient medical records or image data. These limitations may have potentially affected the outcomes observed. In addition, dosimetry analysis and evaluation of quality of life were not included in the present study. However, all of the patients in the present cohort received platinumbased chemotherapy and popular treatment modalities, and a potential benefit of IMRT replanning associated with an actual clinical advantage of local control for T4 stage NPC was observed.

In conclusion, the results of the present study demonstrate that IMRT can provide excellent local-regional control for T4 stage NPC, and IMRT replanning may further improve local control. The incorporation of GTVln into the N staging system may also provide better prognostic information. With distant metastasis observed to be the major cause of treatment failure in the present study, treatment modalities that effectively reduce the rate of distant metastasis and increase the survival rate of T4 stage NPC patients still need to be explored.

\section{Competing Interests}

The authors declare that they have no competing interests.

\section{Authors' Contributions}

Yangkun Luo and Yang Gao contributed equally to this paper.

\section{References}

[1] A. T. C. Chan, P. M. L. Teo, and P. J. Johnson, "Nasopharyngeal carcinoma," Annals of Oncology, vol. 13, no. 7, pp. 1007-1015, 2002.

[2] A. T. C. Chan, S. F. Leung, R. K. C. Ngan et al., "Overall survival after concurrent cisplatin-radiotherapy compared with radiotherapy alone in locoregionally advanced nasopharyngeal carcinoma," Journal of the National Cancer Institute, vol. 97, no. 7, pp. 536-539, 2005. 
[3] S. B. Edge and C. C. Compton, "The American Joint Committee on Cancer: the 7th edition of the AJCC cancer staging manual and the future of TNM," Annals of Surgical Oncology, vol. 17, no. 6, pp. 1471-1474, 2010.

[4] M. K. M. Kam, P. M. L. Teo, R. M. C. Chau et al., “Treatment of nasopharyngeal carcinoma with intensity-modulated radiotherapy: the Hong Kong experience," International Journal of Radiation Oncology Biology Physics, vol. 60, no. 5, pp. 14401450, 2004.

[5] N. Lee, J. Harris, A. S. Garden et al., "Intensity-modulated radiation therapy with or without chemotherapy for nasopharyngeal carcinoma: radiation therapy oncology group phase ii trial 0225," Journal of Clinical Oncology, vol. 27, no. 22, pp. 36843690, 2009.

[6] X. Sun, S. Su, C. Chen et al., "Long-term outcomes of intensitymodulated radiotherapy for 868 patients with nasopharyngeal carcinoma: an analysis of survival and treatment toxicities," Radiotherapy and Oncology, vol. 110, no. 3, pp. 398-403, 2014.

[7] W. K. Bae, J. E. Hwang, H. J. Shim et al., "Phase II study of docetaxel, cisplatin, and 5-FU induction chemotherapy followed by chemoradiotherapy in locoregionally advanced nasopharyngeal cancer," Cancer Chemotherapy and Pharmacology, vol. 65, no. 3, pp. 589-595, 2010.

[8] L. Chen, C.-S. Hu, X.-Z. Chen et al., "Concurrent chemoradiotherapy plus adjuvant chemotherapy versus concurrent chemoradiotherapy alone in patients with locoregionally advanced nasopharyngeal carcinoma: a phase 3 multicentre randomised controlled trial," The Lancet Oncology, vol. 13, no. 2, pp. 163-171, 2012.

[9] G. Fountzilas, E. Ciuleanu, M. Bobos et al., "Induction chemotherapy followed by concomitant radiotherapy and weekly cisplatin versus the same concomitant chemoradiotherapy in patients with nasopharyngeal carcinoma: a randomized phase II study conducted by the Hellenic Cooperative Oncology Group (HeCOG) with biomarker evaluation," Annals of Oncology, vol. 23, no. 2, pp. 427-435, 2012.

[10] E. P. Hui, B. B. Ma, S. F. Leung et al., "Randomized phase II trial of concurrent cisplatin-radiotherapy with or without neoadjuvant docetaxel and cisplatin in advanced nasopharyngeal carcinoma," Journal of Clinical Oncology, vol. 27, no. 2, pp. 242-249, 2009.

[11] A. W. M. Lee, S. Y. Tung, D. T. T. Chua et al., "Randomized trial of radiotherapy plus concurrent-adjuvant chemotherapy vs radiotherapy alone for regionally advanced nasopharyngeal carcinoma," Journal of the National Cancer Institute, vol. 102, no. 15, pp. 1188-1198, 2010.

[12] A. M. Chen, M. E. Daly, J. Cui, M. Mathai, S. Benedict, and J. A. Purdy, "Clinical outcomes among patients with head and neck cancer treated by intensity-modulated radiotherapy with and without adaptive replanning," Head and Neck, vol. 36, no. 11, pp. 1541-1546, 2014.

[13] H. Yang, W. Hu, W. Wang, P. Chen, W. Ding, and W. Luo, "Replanning during intensity modulated radiation therapy improved quality of life in patients with nasopharyngeal carcinoma," International Journal of Radiation Oncology Biology Physics, vol. 85, no. 1, pp. e47-e54, 2013.

[14] W. Wang, H. Yang, W. Hu et al., "Clinical study of the necessity of replanning before the 25th fraction during the course of intensity-modulated radiotherapy for patients with nasopharyngeal carcinoma," International Journal of Radiation Oncology, Biology, Physics, vol. 77, no. 2, pp. 617-621, 2010.
[15] T. Nishi, Y. Nishimura, T. Shibata, M. Tamura, N. Nishigaito, and M. Okumura, "Volume and dosimetric changes and initial clinical experience of a two-step adaptive intensity modulated radiation therapy (IMRT) scheme for head and neck cancer," Radiotherapy and Oncology, vol. 106, no. 1, pp. 85-89, 2013.

[16] L. Zhao, Q. Wan, Y. Zhou, X. Deng, C. Xie, and S. Wu, “The role of replanning in fractionated intensity modulated radiotherapy for nasopharyngeal carcinoma," Radiotherapy and Oncology, vol. 98, no. 1, pp. 23-27, 2011.

[17] ICRU, "Prescribing, recording, and reporting photon beam therapy," Tech. Rep. 50, International Commission on Radiation Units and Measurements, Bethesda, Md, USA, 1993.

[18] International Commission on Radiation Units and Measurements, "Report 62: prescribing, recording and reporting photon beam therapy," Supplement to ICRU Report 50, International Commission on Radiation Units and Measurements, Bethesda, Md, USA, 1999.

[19] Institute NC, Common Terminology Criteria for Adverse Events v3. 0 (CTCAE), Cancer Therapy Evaluation Program, 2006.

[20] J. D. Cox, J. Stetz, and T. F. Pajak, "Toxicity criteria of the Radiation Therapy Oncology Group (RTOG) and the European organization for research and treatment of cancer (EORTC)," International Journal of Radiation Oncology, Biology, Physics, vol. 31, no. 5, pp. 1341-1346, 1995.

[21] L. Chen, L.-Z. Liu, M. Chen et al., "Prognostic value of subclassification using MRI in the T4 classification nasopharyngeal carcinoma intensity-modulated radiotherapy treatment," International Journal of Radiation Oncology Biology Physics, vol. 84, no. 1, pp. 196-202, 2012.

[22] C.-N. Cao, J.-W. Luo, L. Gao et al., "Update report of T4 classification nasopharyngeal carcinoma after intensity-modulated radiotherapy: an analysis of survival and treatment toxicities," Oral Oncology, vol. 51, no. 2, pp. 190-194, 2015.

[23] N. Lee, P. Xia, J. M. Quivey et al., "Intensity-modulated radiotherapy in the treatment of nasopharyngeal carcinoma: an update of the UCSF experience," International Journal of Radiation Oncology, Biology, Physics, vol. 53, no. 1, pp. 12-22, 2002.

[24] S. Lin, J. J. Lu, L. Han, Q. Chen, and J. Pan, "Sequential chemotherapy and intensity-modulated radiation therapy in the management of locoregionally advanced nasopharyngeal carcinoma: experience of 370 consecutive cases," BMC Cancer, vol. 10, article 39, 2010.

[25] F. C. S. Wong, A. W. Y. Ng, V. H. F. Lee et al., "Whole-field simultaneous integrated-boost intensity-modulated radiotherapy for patients with nasopharyngeal carcinoma," International Journal of Radiation Oncology Biology Physics, vol. 76, no. 1, pp. 138-145, 2010.

[26] Y.-P. Mao, S.-B. Liang, L.-Z. Liu et al., “The N staging system in nasopharyngeal carcinoma with radiation therapy oncology group guidelines for lymph node levels based on magnetic resonance imaging," Clinical Cancer Research, vol. 14, no. 22, pp. 7497-7503, 2008.

[27] A. W. M. Lee, W. T. Ng, L. L. K. Chan et al., "Evolution of treatment for nasopharyngeal cancer-success and setback in the intensity-modulated radiotherapy era," Radiotherapy and Oncology, vol. 110, no. 3, pp. 377-384, 2014.

[28] M. Lan, Y. Huang, C. Y. Chen et al., "Prognostic value of cervical nodal necrosis in nasopharyngeal carcinoma: analysis of 1800 patients with positive cervical nodal metastasis at MR imaging," Radiology, vol. 276, no. 2, pp. 536-544, 2015. 
[29] L.-L. Tang, R. Guo, G. Zhou et al., "Prognostic value and staging classification of retropharyngeal lymph node metastasis in nasopharyngeal carcinoma patients treated with intensitymodulated radiotherapy," PLoS ONE, vol. 9, no. 10, Article ID e108375, 2014.

[30] L. Y. Zheng, S. X. Yan, D. F. Yan, J. S. Yang, and Y. X. Wang, "Fatal bleeding in a nasopharyngeal carcinoma patient after concurrent chemoradiation plus cetuximab: a case report," OncoTargets and Therapy, vol. 6, pp. 703-706, 2013.

[31] J.-X. Wu, L.-Y. Xu, B.-H. Yang et al., "Clinical analysis of 60 cases with radiative nasopharyngeal necrosis in nasopharyngeal carcinoma," Zhonghua Er Bi Yan Hou Tou Jing Wai Ke Za Zhi, vol. 47, no. 3, pp. 185-190, 2012.

[32] S.-Z. Lai, W.-F. Li, L. Chen et al., "How does intensitymodulated radiotherapy versus conventional two-dimensional radiotherapy influence the treatment results in nasopharyngeal carcinoma patients?" International Journal of Radiation Oncology, Biology, Physics, vol. 80, no. 3, pp. 661-668, 2011.

[33] Y. Song, W. Wang, G. Tao, and X. Zhou, "Survival benefit of induction chemotherapy in treatment for locally advanced nasopharyngeal carcinoma-a time-to-event meta-analysis," Oral Oncology, vol. 51, no. 8, pp. 764-769, 2015.

[34] M. Yan, A. Kumachev, L. L. Siu, and K. K. W. Chan, "Chemoradiotherapy regimens for locoregionally advanced nasopharyngeal carcinoma: a Bayesian network meta-analysis," European Journal of Cancer, vol. 51, no. 12, pp. 1570-1579, 2015.

[35] P. Blanchard, A. Lee, S. Marguet et al., "Chemotherapy and radiotherapy in nasopharyngeal carcinoma: an update of the MAC-NPC meta-analysis," The Lancet Oncology, vol. 16, no. 6, pp. 645-655, 2015. 


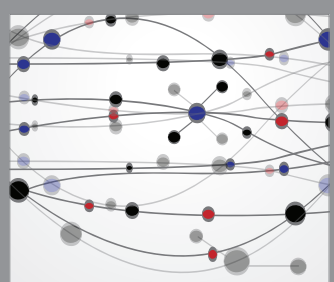

The Scientific World Journal
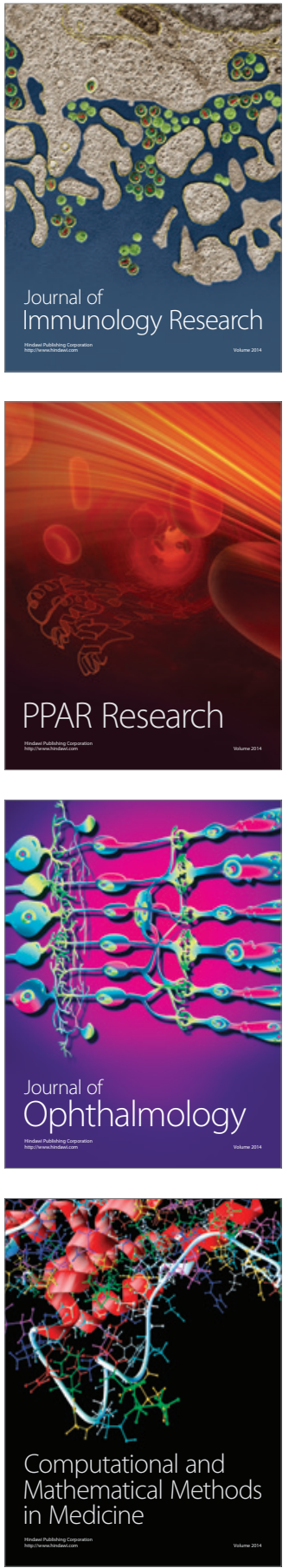

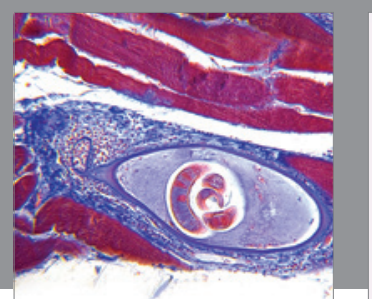

Gastroenterology Research and Practice

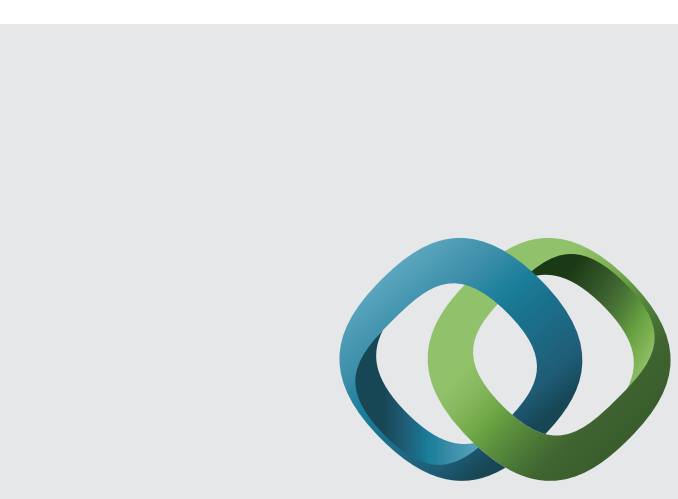

\section{Hindawi}

Submit your manuscripts at

http://www.hindawi.com
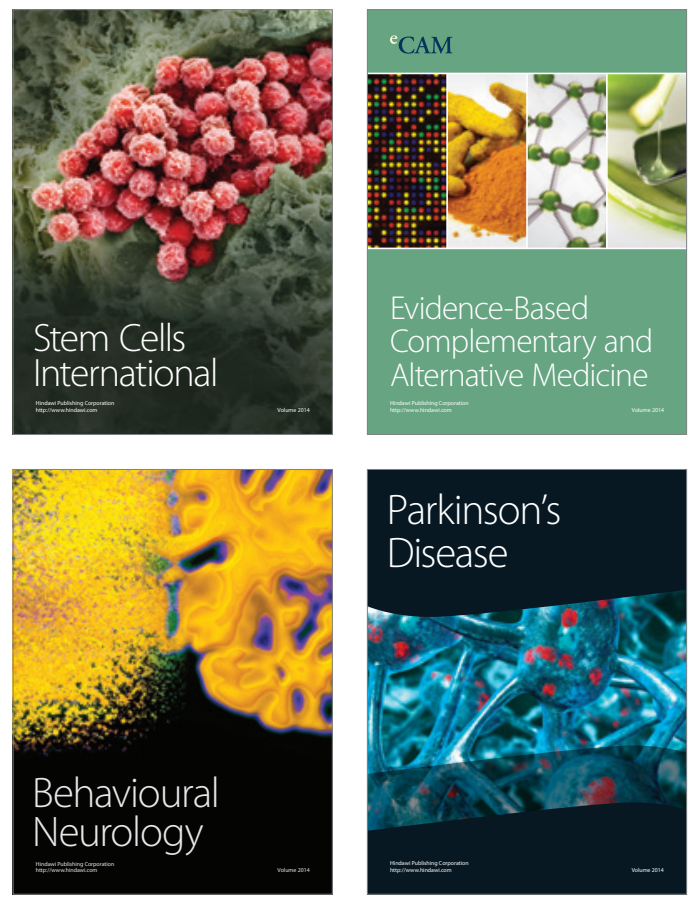
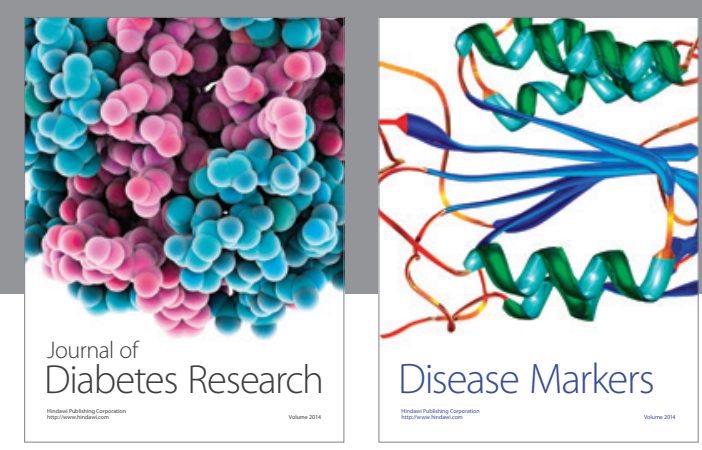

Disease Markers
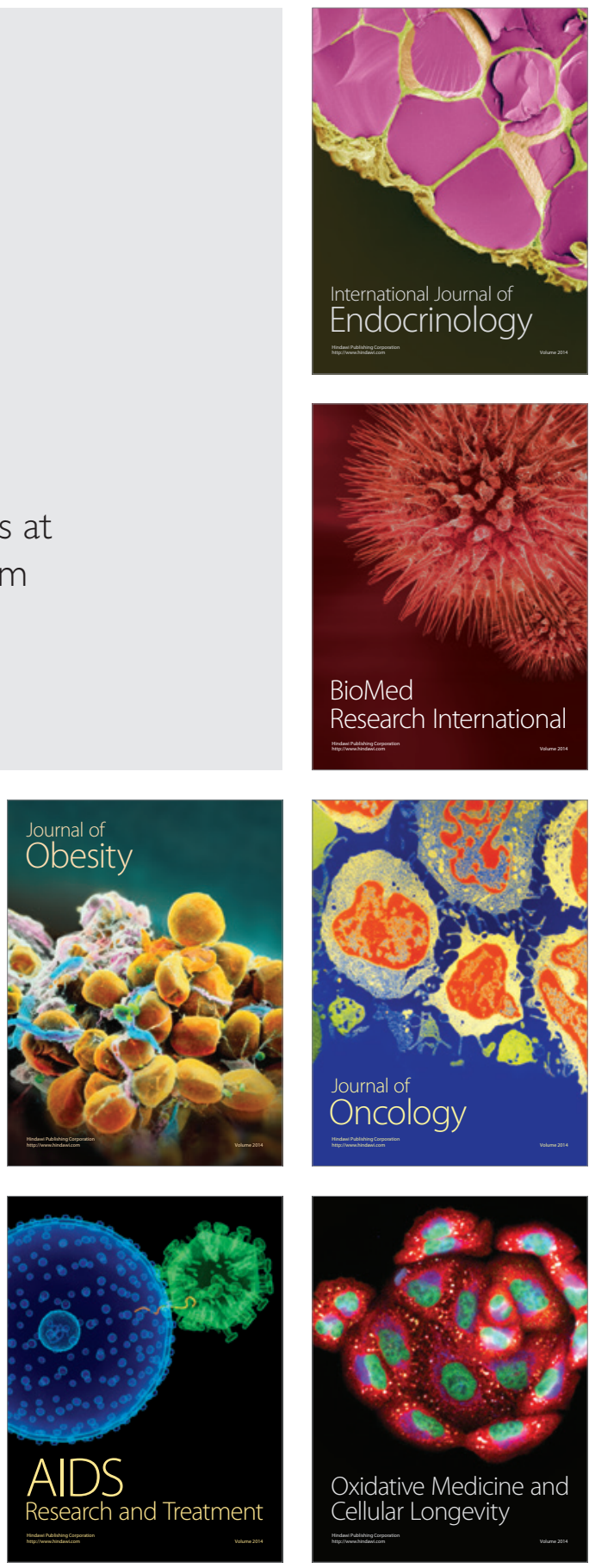